\title{
Epidemiological survey of chronic diseases on manufacturing employees (ESCOME): Design and methodology of a large cross-sectional study
}

\author{
Hamidreza Roohafza ${ }^{\mathrm{a}}$, Awat Feizi ${ }^{\mathrm{a}, \mathrm{b}, *}$, Shahnaz Amani Tirani ${ }^{\mathrm{c}}$, Katayoun Rabiei ${ }^{\mathrm{d}}$, \\ Ahmad Bahonar ${ }^{\mathrm{e}}$, Hassan Alikhasi ${ }^{\mathrm{d}}$, Nizal Sarrafzadegan ${ }^{\mathrm{d}}$ \\ ${ }^{\text {a } C a r d i a c ~ R e h a b i l i t a t i o n ~ R e s e a r c h ~ C e n t e r, ~ C a r d i o v a s c u l a r ~ R e s e a r c h ~ I n s t i t u t e, ~ I s f a h a n ~ U n i v e r s i t y ~ o f ~ M e d i c a l ~ S c i e n c e s, ~ I s f a h a n, ~ I r a n ~}$ \\ ${ }^{\mathrm{b}}$ Department of Biostatistics and Epidemiology, School of Health, Isfahan University of Medical Sciences, Isfahan, Iran \\ ${ }^{\mathrm{c}}$ Khorshid Hospital, Nutrition Department, Isfahan University of Medical Sciences, Isfahan, Iran \\ ${ }^{\mathrm{d}}$ Isfahan Cardiovascular Research Center, Cardiovascular Research Institute, Isfahan University of Medical Sciences, Isfahan, Iran \\ ${ }^{\mathrm{e}}$ Hypertension Research Center, Cardiovascular Research Institute, Isfahan University of Medical Sciences, Isfahan, Iran
}

\section{A R T I C L E I N F O}

\section{Keywords:}

Epidemiology

Chronic diseases

Life style

Job-related variables

Occupational health

Manufacturing employees

\begin{abstract}
A B S T R A C T
Background and objectives: The epidemiological survey of chronic diseases on manufacturing employees (ESCOME) was designed to a) assess the prevalence of chronic physical and mental diseases b) investigate sociodemographic, psychological and lifestyle determinants of chronic physical and mental diseases c) investigate job-related determinants of chronic physical and mental diseases among a large sample of Esfahan steel company (ESCO) employees.

Methods: This cross-sectional study was conducted at 2015 in two main phases among employees of ESCO, Isfahan, Iran. In the first phase, validated self-administered questionnaires were used to assess quality of life, mental health, lifestyle and job-related variables. In the second phase, clinical, biomedical and anthropometric indices were evaluated in the occupational medicine center of ESCO. Furthermore, a self-report questionnaire was used to collect comprehensive information about socio-demographic factors, smoking habits and chronic physical diseases.

Results: Totally, 3063 employees from various departments of ESCO were recruited in the current study. The participants' mean age was 36.74 years (SD $=7.30$, ranged from 21 to 64 ) and $91.5 \%$ of them were male. The participants predominantly worked in rotational shifts (55\%) and 63.5\% were low-level blue-collar employees. Hyperlipidemia (29.7\%) and anxiety (8.9\%) were the most prevalent physical and mental diseases respectively. Conclusions: The ESCOME provided an opportunity to enhance our knowledge about the epidemiology and determinants of chronic physical and mental diseases among Iranian manufacturing employees.
\end{abstract}

\section{Introduction}

According to the 2015 World Health Organization report (WHO), every year more than one third of deaths due to chronic diseases happen in working-age population mainly in low and middle income countries. ${ }^{1}$ Previous investigations have suggested that chronic physical and mental diseases imposing a substantial economic burden to industry because of productivity loss and employees' absence. ${ }^{2-4}$ It has also been reported that workplace health promotion programs targeting nutrition and physical activity improve work performance, presenteeism and productivity. ${ }^{5,6}$ The impact of health status on financial outcome of industries is more important in developing countries with rapid economic growth. Thus, it is a necessitate to investigate the prevalence and determinants of chronic diseases in workforce population in these countries.

Some modifiable risk factors such as smoking, physical inactivity and unhealthy diet are known as key risk factors of prevalent chronic diseases. However, in recent years there has been an increasing interest in detecting the association between job-related factors with risk of chronic diseases. Several review studies have suggested that there is an association between job-related variables and risk of chronic diseases such as cardiovascular disorders, breast cancer and psychological diseases. $^{7-9}$ Although, undertaking further studies are recommended considering the large heterogeneity associated with differences in design of studies. Additionally, to design effective workplace health promotion interventions, it is necessary to explore job-related factors of

\footnotetext{
* Corresponding author. Cardiac Rehabilitation Research Center, Cardiovascular Research Institute, Isfahan University of Medical Sciences, Isfahan, Iran.

E-mail address: awat_feiz@hlth.mui.ac.ir (A. Feizi).
} 
mental and physical diseases and their interrelationship with lifestyle and socio-demographic factors in different workforce populations. The ESCOME is a comprehensive cross-sectional study which was designed to a) assess the prevalence of chronic physical and mental diseases b) investigate socio-demographic, psychological and lifestyle determinants of chronic physical and mental diseases c) investigate job-related determinants of chronic physical and mental diseases among a large sample of Esfahan steel company (ESCO) employees.

\section{Methods}

\subsection{Study design and participants' recruitment}

This cross-sectional study was conducted at 2015 among 16,000 formal and contractual employees of Esfahan Steel Company (ESCO). Sample size in the current epidemiological study was determined in order to achieve an accurate and reliable estimate of the prevalence of psychological disorders. The sample size was estimated to be 3500 with $0.1,0.05$ and 0.01 to be the values for the prevalence of psychological problems, type one error rate and sampling error rate respectively. ${ }^{10,11}$

The inclusion criteria were work experience for at least one year and willing and agreeing to participate in the study. The participants who did not answer to a large fraction of questions (more than $10 \%$ of the questionnaires' pages) were excluded from analyses. Multi-stage cluster sampling along with stratified sampling based on managerial sections were employed for participants' selection. Clusters were seven main management departments and their subsidiary sections and the strata were job categories of employees in ESCO. The sample size in each cluster and strata was proportional to size. Due to random nature of sampling, as well as low number of female employees $(n=800)$, in order to have sufficient number of women participants in our study, 260 females who agreed to participate in the study were included using convenience sampling. The data gathering tools were self-administrated questionnaires. The data from 3063 (response rate: 0.87) participants who had completed questionnaires was used. Employees in managerial and supervisory positions were considered as high-level white-collar employees and office employees were considered as low-level white collar employees. Blue collar employees were also classified as highlevel (skilled workers) and low-level (semi-skilled and non-professional workers). Eligible people received a letter explaining the objectives of the study and inviting for collaboration. Medical research ethics committee of the Cardiovascular Research Institute affiliated to Isfahan University of Medical Sciences approved the final study protocol (Research project number: 87115) and informed consent was obtained from all participants before participating in the study.

\subsection{Study phases}

\subsubsection{First phase}

In the first phase of the study, different validated self-administered questionnaires on quality of life, mental health, lifestyle and job-related variables distributed among ESCO employees in various departments. The process of questionnaires administration and data collection was conducted during 6 months at the company and research assistances guided the participants regarding the possible ambiguities and simultaneously the process was monitored rigorously by study coordinators over the study period. Each questionnaire described in detail below.

International Physical Activity Questionnaire (IPAQ): International physical activity questionnaire short form (IPAQ-SF) has been developed to assess physical activity in cross-sectional studies. ${ }^{12}$ The recall period of the questionnaire is the last 7 days and four types of physical activities are recorded including, vigorous activities such as aerobics, moderate-intensity activities such as leisure cycling, walking and sitting. In the current study, we used the validated Persian form of IPAQ-SF with Cronbach's $\alpha$ of $0.87 .{ }^{13}$ Participants were asked about both the frequency (the number of days that they had been involved in the physical activity) and the duration (the average time they had spent on the physical activity in minute) of their physical activities in the questionnaire. The sum of scores for different types of physical activities was considered as total physical activity score.

Food frequency questionnaire (FFQ-SF): A self-administered food frequency questionnaire (48-item FFQ), was used to assess dietary data of the industrial employees. The 48-item FFQ consists of a list of foods and food groups commonly consumed by Iranians people. The FFQshort form has been classified into different foods and food groups including, grains (bread, rice, different types of 'cakes, pastry and biscuits', potato, spaghetti, soya, beans), dairy products (low fat milk, low fat yogurt, high fat milk, high fat yogurt, butter, cream, cheese, Dugh), fruits, vegetables, oils (liquid oil, hydrogenated oil), meats ('liver-lung', processed meats, poultry, red meat, fish), mixed dishes (canned food, fast foods, fried foods and restaurant's foods, Kale Pache), and miscellaneous food items and beverages (including carbonated drinks, all kinds of jam, fresh fruit juice, all kinds of seeds, fruit juice, mayonnaise, egg, nuts, garlic).

Firstly, participants were asked to select "yes" option if they consumed the proposed food items. Then, based on "yes" answer, they were asked to recall the duration of food consumption as daily, weekly, monthly or yearly basis in an open-ended format. Finally, according to previous answer, participants were asked to report frequency of food consumption as 'times' per each day, or week or month or year. The reported frequency of each food item was then converted into a weekly consumption ('times' of consumption in a week). Participants also could select seldom/never option if they never consumed the proposed food items. Seldom and never items were considered as "zero." 48-item version of FFQ was validated for Iranian population. Reproducibility of the FFQ was assessed by intra class correlation coefficient (ICC) and it was found to be between 0.47 and 0.69 in different food groups. ${ }^{14}$

Stressful Life Event questionnaire (SLE): The stressful life event questionnaire is a 44- item self -report instrument that assesses the intensity of stress both in general population and primary care settings. The questionnaire includes 11 subscales: home life, financial problems, social relation, personal conflicts, job conflicts, educational concerns, job security, loss and separation, sexual life, daily life and health concerns. The response format was a five-point Likert scale ranged from very mild to very severe. The Iranian version of SLE was applied in the present study. ${ }^{15}$ The Persian version showed moderate significant correlation coefficient among domains of the SLE questionnaire and GHQ12 score. The results of the discriminate validity analysis were acceptable statistically. In addition, internal validity based on Cronbach'alpha coefficient was found to be 0.92 .

Effort Reward Imbalance questionnaire (ERI): The effort-reward imbalance (ERI) model has been developed to identify stressful work features. ${ }^{16}$ The 23 -item ERI questionnaire is composed of 3 scales including, effort (6 items), reward (11 items) and over-commitment (6 items). In the effort and reward scales, participants were asked to answer to statements which imply typical experiences in their work situation on a five-point Likert scale where a value of 1 indicates no respective stressful experience, whereas a value of 5 indicates very high stressful experience. However, the over-commitment scale items were assessed using a four-point Likert scale ranging from strongly disagree to strongly agree. The Persian version of ERI has been validated in a group of Iranian workers. The Cronbach's $\alpha$ coefficients for effort, reward and over-commitment subscales have been obtained as $0.61,0.85$ and 0.67 , respectively. ${ }^{17} \mathrm{~A}$ ratio between the two scales effort and reward was calculated to define ERI. Participants who had an effort/reward ratio score of 1.0 or higher have been considered to experience ERI.

General Health Questionnaire-12 (GHQ-12): The questionnaire is a screening tool that is widely used to detect minor psychiatric morbidity in community and primary care settings. ${ }^{18}$ In the current study the Persian version of the 12-item general health questionnaire (GHQ- 
12) with a bi-modal scoring style (0-0-1-1) was used. ${ }^{19}$ The final score ranged from 0 to 12 and a cut off score of 4 or more points was considered as affecting by psychological distress. Reliability analysis the Persian version showed satisfactory Cronbach's alpha coefficient $=0.87$. Convergent validity indicated a significant negative correlation between the GHQ-12 and global quality of life scores. The factor analysis conducted for evaluation construct validity showed that the GHQ-12 was a measure of psychological morbidity with two-factor structure including psychological distress' and 'social dysfunction' that jointly accounted for $51 \%$ of the variance.

Hospital Anxiety and Depression Scale (HADS): The instrument is a brief, self-administered scale for identification of anxiety and depression among general population, psychiatric and primary care patients. ${ }^{20}$ The Persian version of the hospital anxiety and depression scale (HADS) with satisfactory psychometric properties was used in the current study. ${ }^{21}$ Cronbach's alpha coefficient has been found to be 0.78 and 0.86 for the HADS anxiety and depression sub-scales, respectively. Validity assessment through groups comparison analysis showed satisfactory results. Both anxiety and depression sub-scales discriminated well between sub-groups of patients differing in clinical status as defined by their disease stage. The 14-item questionnaire composed of two seven-item subscales for anxiety and depression. Items in each subscale are scored on a 4- point Likert type scale (0-1-2-3); thus, the total score ranges from 0 to 21 in each subscale. A total score of 11 or higher indicates considerable symptoms of depression and anxiety.

Euro Quality of life-5-dimension questionnaire (EQ-5D): The Persian version of Euro quality of life-5-dimension questionnaire (EQ5D) was applied to evaluate quality of life among participants. The instrument has become very popular because of ease in its administration, scoring and interpretation. In addition, its psychometric properties are equivalent with other comprehensive instruments. ${ }^{22,23}$ The EQ-5D score was able to differentiate patients according to the severity of the disease based on the EQ-5D VAS ( $p<0.0001$ ). The ICC for EQ5D and EQ-5D VAS were 0.753 and 0.896 , respectively. The correlation of total score of the EQ-5D questionnaire with the total scores of SF-36 was $(r=0.445)$ and dimensions of EQ-5D questionnaire with counterpart dimensions from SF-36 questionnaire were found to be $(r=0.37-0.45)$ indicating a moderate correlation. ${ }^{24}$ Basically, EQ-5D contains two modules including, EQ-5D self-report questionnaire and EQ-5D visual analogue scale (VAS). The EQ-5D self-report questionnaire consists of 5 dimensions (mobility, self-care, usual activities, pain/discomfort, and anxiety/depression) and three levels in each dimension including, no problems, some problems, and severe problems. Participants were asked to choose a response that best describes their current situation in each domain. The total score of the questionnaire was defined by the sum of dimensions' scores and higher scores of EQ5D indicated poor QOL. They also rated their overall health on a VAS ranging from 0 to 100 .

\subsubsection{Second phase}

In the second phase of the study, participants were referred to occupational medicine center of ESCO to assess various anthropometric, clinical and biochemical indices. A self-report questionnaire was also distributed among participants to assess socio-demographic factors, smoking habits and chronic physical disorders. The socio-demographic factors included age, gender, marital status (married, single) and educational levels $(0-5,6-12$ and $>12$ years). Participants were also asked if they have ever smoked cigarette and those participants who answered yes were asked whether they smoke cigarettes currently and the number of cigarettes they smoke. The same questions were also asked about hookah habits. Participants reported their history about physical chronic diseases including hypertension, diabetes, hyperlipidemia, malignancies, stroke, coronary artery diseases and respiratory diseases.

Anthropometrics: Anthropometric indices were measured according to standard protocols by a trained medical staff. Weight was measured in kilogram $(\mathrm{kg})$ in light clothing without shoes and recorded to the nearest $0.5 \mathrm{~kg}$. Height was measured to the nearest $0.1 \mathrm{~cm}$ using a stadiometer without shoes and BMI was computed as the ratio of weight $(\mathrm{kg})$ to the square of height $\left(\mathrm{m}^{2}\right)$. A tape measure was used to measure waist circumference midway between the lowest rib and the iliac crest while the participants were standing. Hip circumference was measured at the point with the maximum size of the buttocks.

Biochemical variables and blood pressure assessment: Overnight fasting blood samples were obtained from participants and collected in ethylenediaminetetra-acetic acid (EDTA) tubes. Then blood samples were centrifuged to separate plasma and serum samples were stored in $-80 \mathrm{C}$ for further analyses. All biochemical tests were done in duplicate in the laboratory of occupational medicine center of ESCO. The concentration of serum lipids (total cholesterol, high density lipoprotein-cholesterol (HDL cholesterol), low density lipoprotein-cholesterol (LDL cholesterol) and triglyceride (TG)) were measured by a commercial kit (Pars Azmoon, Iran). The level of fasting blood glucose (FBS) was also measured using Biosystems kit (France). Blood pressure was measured twice in each arm in the sitting position using a standard mercury sphygmomanometer by a trained operator. To increase the reliability of biochemical variables, each value was assessed by two independent assessors and its average was considered for analyses. There was a 5-min interval between each two measurements and the mean of two values was used for analysis.

\subsection{Data quality assurance}

The validity of the data was evaluated at different stages. Project's managerial coordinator verified $5 \%-10 \%$ of the questionnaires weekly; and those questionnaires with more than $10 \%$ of un-completed pages, were excluded. The data sheets were checked by an independent researcher and $10 \%$ of entered data into statistical package was matched with the questionnaires by another researcher, independently. Although the inclusion and exclusion criteria were assessed at the beginning of the study, they were rechecked on the computerized data. After assessing missing values and outlier items, the total score of scale and subscales were calculated based on the valid instructions of each questionnaire. In addition, new variables from raw data sheets were established based on the study's objectives. The duration of data handling was lasted about 6 months.

\subsection{Statistical analysis}

In this paper, quantitative and categorical data were presented as mean (SD) ([range: minimum-maximum]), and frequency (percentage), respectively. Data analyses were performed using Statistical Package for Social Sciences version 16 (SPSS Inc., Chicago, IL, U.S.A.).

\subsection{Plan for future data analyses}

According to objectives of the project, various statistical methods such as independent samples student $t$-test, Chi-square test, analysis of variance (ANOVA), analysis of covariance (ANCOVA), correlation and univariate, multivariable, multivariate linear regression and logistic regression analyses will be applied. Also, factor analysis, latent class analysis, and mixture factor models will be used for group and variables separation proposes. According to the structure of the collected data in this study, i.e. hierarchical (multilevel), in which employees as the level-1 units are nested within different managerial departments, as the level-2 units, advanced multilevel analyses such as linear mixed effects models, multilevel latent class model, multilevel mixture factors model, multi-group factor models and multilevel structural equation modeling are applicable in the data analysis. 
Table 1

Basic demographic, lifestyle, job related and clinical characteristics of study participants.

\begin{tabular}{ll}
\hline Variables & $\begin{array}{l}\text { Number }(\%) \text { or mean } \pm \text { SD (minimum- } \\
\text { maximum) }\end{array}$ \\
\hline Basic and lifestyle variables & \\
\hline Age (year) & $36.74 \pm 7.30$ \\
Gender & \\
Male & $2803(91.5)$ \\
Female & $260(8.5)$ \\
Marital Status & \\
Married & $2758(90)$ \\
Single & $305(10)$ \\
Education & \\
0-5 years & $255(8.3)$ \\
6-12 years & $1908(62.3)$ \\
$>12$ years & $900(29.4)$ \\
Smoking status & \\
Cigarette & \\
Never & $2171(70.9)$ \\
Former & $620(20.2)$ \\
Current & $272(8.9)$ \\
Hookah & \\
Never & $2725(89)$ \\
Former & $228(7.4)$ \\
Current & $110(3.6)$ \\
Physical activity(h/week) & $7.33 \pm 3.65(1-30.14)$ \\
Sleep duration(h) & \\
\hline
\end{tabular}

Physical examination variables

Blood pressure

Systolic BP (mmHg)

Diastolic BP (mmHg)

Anthropometric data

Body mass index $\left(\mathrm{kg} / \mathrm{m}^{2}\right)$

Waist circumference $(\mathrm{cm})$

Hip circumference $(\mathrm{cm})$

Waist/Hip ratio

Biochemical data

Fasting blood sugar $(\mathrm{mg} / \mathrm{dL})$

Total cholesterol (mg/dL)

High density lipoprotein cholesterol (mg/dL)

Low density lipoprotein cholesterol (mg/dL)

Triglyceride $(\mathrm{mg} / \mathrm{dL})$

Blood Urea Nitrogen (mg/dL)

Creatinine (mg/dL)

Job related variables

Shift work

No

Second Job

Yes

No

Job stress

Effort

Reward

Over-commitment

ER ratio*

Occupational Categories

High-level white collar

Low-level white collar

High-level blue collar

Low-level blue collar

$16.9 \pm 11.0(80-185.0)$

$77.7 \pm 8.4(50-120)$

$25.58 \pm 3.80(15.30-45.49)$

$88.61 \pm 9.99(58-130)$

$100.24 \pm 6.94(52-134)$

$0.88 \pm 0.07(0.64-1.75)$

$91.82 \pm 19.41$ (59-344)

$182.73 \pm 35.11(83-350)$

$42.49 \pm 8.06(19-124)$

$92.43 \pm 21.50(38-185)$

$150.25 \pm 99.85(26-118)$

$28.18 \pm 8.01(19-48)$

$1.01 \pm 0.12(0.90-1.20)$
Table 1 (continued)

\begin{tabular}{ll}
\hline Variables & $\begin{array}{l}\text { Number (\%) or mean } \pm \text { SD (minimum- } \\
\text { maximum) }\end{array}$ \\
\hline $\begin{array}{l}\text { Chronic Physical Diseases prevalence } \\
\text { Diabetes mellitus }\end{array}$ & $89(4)$ \\
Hyperlipidemia & $701(29.7)$ \\
High blood pressure & $368(12)$ \\
Psychological Disorders Prevalence & \\
Depression & $202(6.6)$ \\
Anxiety & $272(8.9)$ \\
Psychological distress & $262(8.6)$ \\
\hline
\end{tabular}

* Effort Reward imbalance ratio.

** Visual Analog Scale (range 0-100).

a Mean \pm SD (Minimun-Maximum) for quantitative variables and frequency (percentage) for categorical variables.

\section{Results}

In the first phase of the study, 3063 invited participants completed study's questionnaires. Major socio-demographic, life style and occupational characteristics of study participants as well as the prevalence of chronic physical and mental disorders are shown in Table 1.

The participants' mean age was 36.74 years $(\mathrm{SD}=7.30$, ranged from 21 to 64 ) and $91.5 \%$ were male. The majority of participants were married (90\%), non-smoker (89\% and 70.9\% hookah and cigarette nonsmoker respectively) with an educational level of 6-12 years (62.3\%). As shown in Table 1, 55\% of participants worked in rotational shifts and low-level blue collar employees were the largest fraction of study population.

Table 1 also presents clinical, anthropometric, biochemical and psychological features of participants. The mean value of BMI in the current study was in the overweight category $\left(25-30 \mathrm{~kg} / \mathrm{m}^{2}\right)$. The mean of all biochemical variable was in the normal range except for HDL cholesterol which is possibly associated with some lifestyle factors such as diet and physical activity. Hyperlipidemia was the most prevalent physical disease (29.7\%) followed by high blood pressure (12\%) and diabetes mellitus (4\%). Depression, anxiety and psychological distress were identified in $6.6 \%, 8.9 \%$ and $8.6 \%$ of employees, respectively. Our results also indicated that the mean values of effort-reward ratio for the total sample was 0.66 . More details about basic characteristics of study participants have been presented in relevant subjects' categories in Table 1.

\section{Discussion}

Occupational and environmental health are important concerns in epidemiology and public health particularly among workforces. Noncommunicable diseases (NCDs) with an increasing prevalence in workforce population create significant burden both on health systems and country economies. The economic impact of NCDs is the result of decreasing work productivity, sick absence and early retirements particularly in manufacturing industries workforce population. ${ }^{25,26}$ It would be a challenging issue in low and middle income countries (LMICs) such as Iran with limited amount of financial resources.

A number of job-related factors are known as risk factors for NCDs in workforce population. A systematic review of 12 studies comprising 2313 cases with chronic heart disease (CHD) showed that long working hours is associated with 1.80 -fold increase in CHD risk. Although, a high heterogeneity was observed because of the difference in sample size, adjustments, design, measurement of exposure and outcome of included studies. ${ }^{7}$ An association between long working hours and mental disorders such as depression and anxiety has also been reported in another systematic investigation by Bannai et al. ${ }^{8}$ However, researchers could not find any conclusive association between the risk of chronic disorders like cancers, cardiovascular disorders, diabetes and 
metabolic syndrome with shift work. ${ }^{9}$ Conducting more epidemiological studies are needed for making etiological inferences among this high risk population considering the lack of consistency in the results of previous investigations as well as limited evidence in this field of research over the world particularly in developing countries. ${ }^{27-31}$ Additionally, the optimal use of reliable data on multifactorial components of workers' health comprising organizational, psychosocial and physical work environment variables is necessary to plan an effective organizational intervention among workforce population for intervening an occupational health problem. As a result, the current study was planned to estimate the prevalence of chronic physical and mental diseases, their risk factors and their interrelationship among a large sample of Esfahan steel company (ESCO) employees.

The primary results of our study and the proposed future analyses in responding to the above emerging issues highlighted the following points: first; some physical illnesses such as hypertension, hyperlipidemia and diabetes are rooted in work conditions, in terms of both work organization and workplace psychosocial environment, second; psychosocial illnesses are initiated from organization conditions and work related factors, third the life style health behaviors such as physical activity and nutritional habits have origins in work conditions, fourth the psychosocial work environment-including the quality of life, job stress, flexibility, work and family conflicts are interrelated.

The strength of the ESCOME drives mostly from its comprehensiveness. In the current study, data on a wide variety of important variables of environmental and occupational health field which affect employees' health status have been collected. The assessment of the interrelationship between these variables and their health consequences provides useful knowledge on occupational health. Multilevel modeling approach which will be used in the study data analysis has some advantages including, the proper modeling of clusters in the research design and understanding the statistical dependence between several factors in the same level or different levels that predict chronic disorders among employees and controlling for potential confounding variables. The most important weakness of the current study that should be noted are its cross-sectional design and lacks of data on some biological, chemical, physical, work and environmental determinants of employees' health conditions.

The results of this study provided useful epidemiological data about wide aspects of industrial employees' health in a developing country and determinants of chronic physical and mental diseases. Furthermore, workplace health promotion and prevention interventions can benefit from the results of this study.

\section{Acknowledgement}

This project was funded in part by Isfahan Steel Company and Cardiovascular Research Institute. We are grateful to all volunteers who participated in this study. We would also like to acknowledge head of Isfahan Steel Company and staff of Cardiovascular Research Institute who help us to conduct the study.

\section{Appendix A. Supplementary data}

Supplementary data to this article can be found online at https:// doi.org/10.1016/j.cegh.2019.08.012.

\section{References}

1. Organisation WH. Non Communicable Diseases. Fact Sheet; January 2015.

2. Van den Heuvel SG, Geuskens GA, Hooftman WE, Koppes LL, Van den Bossche SN Productivity loss at work; health-related and work-related factors. J Occup Rehabil 2010;20:331-339.
3. Merrill RM, Aldana SG, Pope JE, et al. Self-rated job performance and absenteeism according to employee engagement, health behaviors, and physical health. $J$ Occup Environ Med. 2013;55:10-18.

4. Kirsten W. Making the link between health and productivity at the workplace-A global perspective. Ind Health. 2010;48:251-255.

5. Pereira MJ, Coombes BK, Comans TA, Johnston V. The impact of onsite workplace health-enhancing physical activity interventions on worker productivity: a systematic review. Occup Environ Med. 2015;72:401-412.

6. Grimani A, Aboagye E, Kwak L. 749 the Impact of Worksite Nutrition and Physical Activity Interventions on Productivity, Work Performance and Work Ability: A Systematic Review. 2018; 2018.

7. Virtanen M, Heikkilä K, Jokela M, et al. Long working hours and coronary heart disease: a systematic review and meta-analysis. Am J Epidemiol. 2012:176:586-596.

8. Bannai A, Tamakoshi A. The association between long working hours and health: a systematic review of epidemiological evidence. Scand $J$ Work Environ Health. 2014;40:5-18.

9. Wang XS, Armstrong ME, Cairns BJ, Key TJ, Travis RC. Shift work and chronic disease: the epidemiological evidence. Occup Med. 2011;61:78-89.

10. Andrea H, Bültmann U, Beurskens AJ, Swaen GM, Van Schayck CP, Kant IJ. Anxiety and depression in the working population using the HAD Scale. Soc Psychiatry Psychiatr Epidemiol. 2004;39:637-646.

11. Sadeghirad B, Haghdoost AA, Amin-Esmaeili M, et al. Epidemiology of major depressive disorder in Iran: a systematic review and meta-analysis. Int J Prev Med. 2010;1:81-91.

12. Bassett Jr DR. International physical activity questionnaire: 12-country reliability and validity. Med Sci Sport Exerc. 2003;35:1396.

13. Kelishadi R, Rabiei K, Khosravi A, et al. Assessment of physical activity of adolescents in Isfahan. J Shahrekord Univ Med Sci. 2001;3:55-66.

14. Mohammadifard N, Sajjadi F, Maghroun M, Alikhasi H, Nilforoushzadeh F N Validation of a simplified food frequency questionnaire for the assessment of dietary habits in Iranian adults: isfahan Healthy Heart Program, Iran. ARYA atheroscler. 2015;11:139-146.

15. Roohafza H, Ramezani M, Sadeghi M, Shahnam M, Zolfagari B, Sarafzadegan N. Development and validation of the stressful life event questionnaire. Int J Public Health. 2011;56:441-448.

16. Siegrist J. Adverse health effects of high-effort/low-reward conditions. J Occup Health Psychol. 1996;1:27-41.

17. Yadegarfar G, Alinia T, Hassannezhad R, et al. Validation and localization of farsi version of effort-reward imbalance questionnaire to measure job stress among employees of isfahan polyacryle corporation. Iran J Epidemiol. 2012;8:73-83.

18. Goldberg D, Williams P, Williams P. A User's Guide to the General Health Questionnaire NferNelson; 1988

19. Montazeri A, Harirchi AM, Shariati M, Garmaroudi G, Ebadi M, Fateh A. The 12-item General Health Questionnaire (GHQ-12): translation and validation study of the Iranian version. Health Oual Life Outcomes. 2003;1:66-69.

20. Bjelland I, Dahl AA, Haug TT, Neckelmann D. The validity of the Hospital Anxiety and Depression Scale: an updated literature review. J Psychosom Res. 2002;52:69-77.

21. Montazeri A, Vahdaninia M, Ebrahimi M, Jarvandi S. The Hospital Anxiety and Depression Scale (HADS): translation and validation study of the Iranian version. Health Qual Life Outcomes. 2003;1:14-18.

22. Gv Willige, Wiersma D, Nienhuis FJ, Jenner JA. Changes in quality of life in chronic psychiatric patients: a comparison between EuroQol (EQ-5D) and WHOQoL. Qual Life Res. 2005; 14:441-451

23. Macran S, Weatherly H, Kind P. Measuring population health: a comparison of three generic health status measures. Med Care. 2003:41:218-231.

24. Dastourani A, Mansour Sohani S, Shah Ali S. Reliability and validity of the Persian version of the european quality of life questionnaire (EQ-5D-3L) in patients with meniscus and knee ligaments injury. J Paramed Sci Rehabil. 2018;7:73-82.

25. Goetzel RZ, Long SR, Ozminkowski RJ, Hawkins K, Wang S, Lynch W. Health, absence, disability, and presenteeism cost estimates of certain physical and mental health conditions affecting US employers. J Occup Environ Med. 2004;46:398-412.

26. Gupta N, Wåhlin-Jacobsen CD, Henriksen LN, Abildgaard JS, Nielsen K, Holtermann A. A participatory physical and psychosocial intervention for balancing the demands and resources among industrial workers (PIPPI): study protocol of a cluster-randomized controlled trial. BMC Public Health. 2015;15:274.

27. Del Duca GF, Silva KS, Garcia LM, de Oliveira ES, Nahas MV. Clustering of unhealthy behaviors in a Brazilian population of industrial workers. Prev Med. 2012;54:254-258.

28. Hosnijeh FS, Christopher Y, Peeters P, et al. Occupation and risk of lymphoid and myeloid leukaemia in the european prospective investigation into cancer and nutrition (EPIC). J Occup Environ Med. 2013;70:464-470.

29. Boshtam M, Sarafzadegan N, Zare K, et al. Effects of 5-year interventions on cardiovascular risk factors of factories and offies employees of isfahan and najafabad: worksite intervention project-isfahan healthy heart program. ARYA atheroscler. 2010;6:94

30. Slottje P, Yzermans CJ, Korevaar JC, Hooiveld M, Vermeulen RC. The populationbased occupational and environmental health prospective cohort study (amigo) in The Netherlands. BMJ open. 2014;4:e005858.

31. Hamra GB, Richardson DB, Cardis E, et al. Cohort profile: the international nuclear workers study (INWORKS). Int J Epidemiol. 2015;45:693-699. 